\title{
Galerkin lumped parameter methods for transient problems
}

\author{
A. Bermúdez ${ }^{* \dagger}$ and F. Pena \\ Departamento de Matemática Aplicada, Universidade de Santiago de Compostela, \\ 15782 Santiago de Compostela, Spain
}

\begin{abstract}
SUMMARY
In this paper we propose a general methodology to obtain lumped parameter models for systems governed by parabolic partial differential equations which we call Galerkin lumped parameter methods. The idea consists of decomposing the computational domain into several subdomains connected through so-called ports. Then a time-independent adapted reduced basis is introduced by numerically solving several elliptic problems in each subdomain. The proposed lumped parameter model is the Galerkin approximation of the original problem in the space spanned by this basis. The relationship of these methods with classical lumped parameter models is analyzed. Numerical results are shown as well as a comparison of the solution obtained with the lumped model and the 'exact' one computed by standard finite element procedures. Copyright (C) 2011 John Wiley \& Sons, Ltd.
\end{abstract}

Received 6 July 2010; Accepted 27 December 2010

KEY WORDS: lumped parameter models; Galerkin approximation; finite element method; heat transfer; reduced basis; domain decomposition

\section{INTRODUCTION}

A lumped parameter model is a simplification in the mathematical model of a physical system where variables that are spatially distributed fields are represented by a finite number of single scalars instead.

A good example of a lumped parameter model is an electrical network, graphically represented by a circuit diagram in which voltages are assigned to the vertices and currents to the edges of the diagram. The mathematical analysis of such a circuit model is much simpler than solving the Maxwell equations for the actual physical system.

Another area where lumped parameter models are frequently used is heat transfer in electric machines arising from energy losses (see for instance [1-6]). This approach has the advantage that solving the model is very fast. However, the developer must invest effort in defining a network that accurately models the main heat-transfer paths. In particular, this process involves determining some capacitances and resistances which are not defined in a precise way.

The goal of this paper is to introduce a systematic methodology called Galerkin lumped parameter method (GLPM) to build lumped parameter models avoiding the previous drawbacks. It consists in viewing these kinds of models as Galerkin approximations of weak formulations of the original distributed parameter problem. A simple idea is to use reduced basis adapted to the particular geometry, materials and physics of the problem. For the sake of simplicity, in this presentation the

\footnotetext{
${ }^{*}$ Correspondence to: A. Bermúdez, Departamento de Matemática Aplicada, Facultad de Matemáticas, Campus Universitario Sur, Universidade de Santiago de Compostela, 15782 Santiago de Compostela, Spain.

†E-mail: alfredo.bermudez@usc.es 
method will be described for heat transfer-like problems. However, the underlying ideas are rather general hence they can be applied to build lumped parameter models in other fields.

Techniques for reduced-order modeling are very popular in the literature. The most well known are proper orthogonal decomposition (POD) (see, for instance, [7]) and reduced basis methods [8-11]. The latter are particularly useful for optimization: taking a set of values for parameters (or 'snapshots') and creating a basis related to them can speed up the search of the optimum. When problems are evolutionary, time is taken as a special parameter.

The approach of these papers is different from ours, where the main goal is to solve timedependent partial differential equations by reducing them to lumped parameters models, i.e. ordinary differential systems involving a small number of unknowns. For instance, the reduced basis method introduced in [8] has a certain resemblance to that presented in this paper. In both methods, the domain is decomposed into smaller subdomains where local bases are calculated by solving steady state boundary-value problems. Then they are glued in order to construct a reduced basis in the original domain. In [8] gluing is done by using mortar techniques hence the global method is not conforming. However, our approach leads to global bases belonging to the space of the weak formulation hence they produce conforming approximations to the solution.

The present paper is organized as follows. In Section 2 we state the time-dependent partial differential equation to be solved. In Section 3, an auxiliary steady-state boundary value problem and the concept of resistance matrix are introduced. In Section 4 the reduced basis is defined and some properties are shown. The original transient problem is considered in Section 5 where a semidiscrete problem associated with the reduced basis (the lumped-parameter model) is introduced and the concept of capacitance is defined. Section 6 combines domain decomposition with the above reduced basis and finally, in Section 7, some numerical results are shown for heat transfer problems in real electric engines.

\section{STATEMENT OF THE PROBLEM}

Let us consider a body $\Omega$ whose boundary $\Gamma$ consists of three parts, $\Gamma=\Gamma^{P} \cup \Gamma^{A} \cup \Gamma^{C}$ each of them possibly having several connected components:

- $\Gamma^{P}=\bigcup_{l=1}^{n^{P}} \Gamma_{l}^{P}$. The $\Gamma_{l}^{P}$ are called the ports of domain $\Omega$. In practical applications they are surfaces in contact with other solid bodies.

- $\Gamma^{C}=\bigcup_{l=1}^{n^{C}} \Gamma_{l}^{C}$. The $\Gamma_{l}^{C}$ are called the convective boundaries of domain $\Omega$.

- $\Gamma^{A}$, to be called the adiabatic boundary of domain $\Omega$.

We assume that the $\Gamma_{i}^{P}, i=1, \ldots, n^{P}$ are the connected components of $\Gamma^{P}$. In other words, two different ports cannot touch each other.

Let us introduce the following transient heat transfer problem in $\Omega$ :

Find the temperature field $\theta(x, t)$ satisfying

$$
\begin{aligned}
\rho c \frac{\partial \theta}{\partial t}-\operatorname{div}(k \operatorname{grad} \theta) & =f \quad \text { in } \Omega \times[0, T], \\
\theta(x, t) & =\theta_{l}^{P}(x, t) \quad \text { on } \Gamma_{l}^{P}, \quad l=1, \ldots, n^{P}, \\
k \frac{\partial \theta}{\partial \mathbf{n}}(x, t)+\alpha_{l}\left(\theta(x, t)-\theta_{l}^{C}(x, t)\right) & =0 \quad \text { on } \Gamma_{l}^{C}, \quad l=1, \ldots, n^{C}, \\
k \frac{\partial \theta}{\partial \mathbf{n}} & =0 \quad \text { on } \Gamma^{A}, \\
\theta(x, 0) & =\theta_{0}(x) \quad \text { in } \Omega,
\end{aligned}
$$


where the data of the problem are

- $\rho$ is the density

- $c$ is the specific heat

- $k$ is the thermal conductivity

- $f$ is the volumetric heating

- $\theta_{l}^{P}(x, t)$ is the temperature at time $t$ and point $x$ of the $l$ th port

- $\theta_{l}^{C}(x, t)$ is the convective temperature at time $t$ and point $x$ of the $l$ th convective boundary

- $\alpha_{l}(x)$ is the time-dependent convective heat transfer coefficient on the lth convective boundary

- $\theta_{0}(x)$ is the initial temperature field.

As it is well known, in order to use a finite element method for space discretization we first introduce a weak formulation by standard procedures (see for instance [12]). Let $\psi$ be a 'test function' defined in $\Omega$ and null on $\Gamma^{P}$. Multiplying equation (1) by $\psi$, integrating in $\Omega$, using a Green's formula, and taking boundary conditions (3) and (4) into account, we get the following problem:

P. Find $\theta(x, t)$ satisfying the Dirichlet boundary conditions (2) and

$$
\begin{aligned}
& \int_{\Omega} \rho c \frac{\partial \theta}{\partial t} \psi \mathrm{d} x+\int_{\Omega} k \operatorname{grad} \theta \cdot \operatorname{grad} \psi \mathrm{d} x+\sum_{l=1}^{n^{C}} \int_{\Gamma_{l}^{C}} \alpha_{l} \theta \psi \mathrm{d} \Gamma \\
& \quad=\int_{\Omega} f \psi \mathrm{d} x+\sum_{l=1}^{n^{C}} \int_{\Gamma_{l}^{C}} \alpha_{l} \theta_{l}^{C} \psi \mathrm{d} \Gamma, \quad \text { for all test function } \psi(x) \text { null on } \Gamma^{P} \\
& \theta(x, 0)=\theta_{0}(x) .
\end{aligned}
$$

Then, we introduce a Galerkin approximation of this problem by using finite elements. Let $\mathscr{T}_{h}$ be a family of meshes of domain $\Omega$ and $V_{h}$ a family of finite element spaces associated with it. We replace the above problem by the semi-discrete approximation:

$\mathbf{P}_{h}$. For $t \in[0, T]$, find $\theta_{h}(., t) \in V_{h}$ satisfying $\theta_{h}(x, t)=\theta_{l h}^{P}(x, t)$ on $\Gamma_{l}^{P}, l=1, \ldots, n^{P}$, and

$$
\begin{aligned}
& \int_{\Omega} \rho c \frac{\partial \theta_{h}}{\partial t} \psi_{h} \mathrm{~d} x+\int_{\Omega} k \operatorname{grad} \theta_{h} \cdot \operatorname{grad} \psi_{h} \mathrm{~d} x+\sum_{l=1}^{n^{C}} \int_{\Gamma_{l}^{C}} \alpha_{l} \theta_{h} \psi_{h} d \Gamma \\
& \quad=\int_{\Omega} f \psi_{h} \mathrm{~d} x+\sum_{l=1}^{n^{C}} \int_{\Gamma_{l}^{C}} \alpha_{l} \theta_{i}^{C} \psi_{h} \mathrm{~d} \Gamma \quad \text { for all } \psi_{h} \in V_{h} \quad \text { null on } \Gamma^{P} \\
& \theta_{h}(x, 0)=\theta_{0 h}(x) \text { in } \Omega,
\end{aligned}
$$

where $\theta_{l h}^{P}$ and $\theta_{0 h}$ are projections of $\theta_{l}^{P}$ and $\theta_{0}$, respectively, on $V_{h}$.

Since $V_{h}$ is of finite dimension, this Galerkin approximation is equivalent to a system of ordinary differential equations which can be solved by numerical discretization methods suitable for stiff problems. In practical applications, the number of unknowns of this system, i.e. the number of degrees of freedom is very large hence the time to solve $\mathbf{P}_{h}$ along a time interval $[0, T]$ can also be very large, making the simulation of changes in a design process very costly.

An alternative approach, which is very popular in some engineering areas such as electromagnetism or thermal analysis of electric motors is to employ the so-called lumped parameters models. The idea is to look for approximate solutions in very low dimension approximation spaces but well adapted to the problem. 


\section{THE STATIONARY PROBLEM. THERMAL RESISTANCE MATRIX}

In order to motivate the choice of the reduced adapted basis to be done in Section 4, let us consider the following stationary problem:

SP.-Given constant values of temperature at the ports, $\theta_{i}^{P}, i=1, \ldots, n^{P}$, and constant convection temperatures, $\theta_{i}^{C}, i=1, \ldots, n^{C}$, at the convective boundaries, find the stationary temperature field $\theta \in H^{1}(\Omega)$ satisfying

$$
\begin{aligned}
-\operatorname{div}(k \operatorname{grad} \theta) & =0 \quad \text { in } \Omega, \\
\theta(x) & =\theta_{i}^{P} \quad \text { on } \Gamma_{i}^{P}, \quad i=1, \ldots, n^{P}, \\
k \frac{\partial \theta}{\partial \mathbf{n}}(x, t)+\alpha_{i}\left(\theta(x, t)-\theta_{i}^{C}\right) & =0 \quad \text { on } \Gamma_{i}^{C}, \quad i=1, \ldots, n^{C}, \\
k \frac{\partial \theta}{\partial \mathbf{n}} & =0 \quad \text { on } \Gamma^{A},
\end{aligned}
$$

where $H^{1}(\Omega)$ is the Sobolev space consisting of the square integrable functions with square integrable gradient.

The weak formulation of this problem can be easily obtained by standard procedures: find $\theta \in H^{1}(\Omega)$ satisfying

$$
\begin{array}{r}
\int_{\Omega} k \operatorname{grad} \theta \cdot \operatorname{grad} \psi \mathrm{d} x+\sum_{l=1}^{n^{C}} \int_{\Gamma_{l}^{C}} \alpha_{l} \theta \psi \mathrm{d} \Gamma=\sum_{l=1}^{n^{C}} \int_{\Gamma_{l}^{C}} \alpha_{l} \theta_{l}^{C} \psi \mathrm{d} \Gamma, \\
\text { for all } \psi \in H^{1}(\Omega) \text { null on } \Gamma^{P}, \\
\theta=\theta_{i}^{P} \quad \text { on } \Gamma_{i}^{P}, \quad i=1, \ldots, n^{P} .
\end{array}
$$

The corresponding heat flows entering into the domain through the $i$ th port and through the $i$ th convective boundary are given by

$$
\begin{aligned}
& Q_{i}^{P}=\int_{\Gamma_{i}^{P}} k \frac{\partial \theta}{\partial \mathbf{n}} \mathrm{d} \Gamma, \quad i=1, \ldots, n^{P}, \\
& Q_{i}^{C}=\int_{\Gamma_{i}^{C}} k \frac{\partial \theta}{\partial \mathbf{n}} \mathrm{d} \Gamma, \quad i=1, \ldots, n^{C},
\end{aligned}
$$

respectively.

It is obvious that the mapping giving these heat fluxes from the temperatures, namely,

$$
\mathbf{K}: \mathbb{R}^{n^{P}+n^{C}} \longrightarrow \mathbb{R}^{n^{P}+n^{C}}
$$

defined by

$$
\mathbf{K}\left(\theta_{1}^{P}, \ldots, \theta_{n^{P}}^{P}, \theta_{1}^{C}, \ldots, \theta_{n^{C}}^{C}\right)=\left(Q_{1}^{P}, \ldots, Q_{n^{P}}^{P}, Q_{1}^{C}, \ldots, Q_{n^{C}}^{C}\right),
$$

is linear; it is also symmetric (see Corollary 3 below).

Moreover, we note that $\mathbf{K}$ is not invertible. In fact, we are going to show that

$$
\operatorname{Ker}(\mathbf{K})=\operatorname{span}\{\mathbf{e}\}
$$

where $\mathbf{e}$ is the vector in $\mathbb{R}^{n^{P}+n^{C}}$ having all components equal to 1 . Indeed, first of all, $\mathbf{K}(\mathbf{e})=\mathbf{0}$ because the solution $\theta$ of problem $\mathbf{S P}$ for $\theta_{1}^{P}=\cdots=\theta_{n^{P}}^{P}=\theta_{1}^{C}=\cdots=\theta_{n^{C}}^{C}=1$ is $\theta(x)=1 \forall x \in \Omega$ hence the corresponding fluxes are null. 
Now, we prove that $\mathbf{K}$ is positive definite on the orthogonal space to $\mathbf{e}$. Let $\left(\theta_{1}^{P}, \ldots, \theta_{n^{P}}^{P}, \theta_{1}^{C}, \ldots\right.$, $\theta_{n}^{C}$ ) be a non-null element in such space and $\theta$ the corresponding solution of (14), (15). Then,

$$
\sum_{i=1}^{n^{P}} \theta_{i}^{P}+\sum_{i=1}^{n^{C}} \theta_{i}^{C}=0
$$

and hence

$$
\theta_{\min }:=\min \left\{\theta_{1}^{P}, \ldots, \theta_{n^{P}}^{P}, \theta_{1}^{C}, \ldots, \theta_{n^{C}}^{C}\right\}<\theta_{\max }:=\max \left\{\theta_{1}^{P}, \ldots, \theta_{n^{P}}^{P}, \theta_{1}^{C}, \ldots, \theta_{n^{C}}^{C}\right\} .
$$

We show, by contradiction, that $\theta$ cannot be constant. Indeed, let us suppose that $\theta$ is constant in $\Omega$. Then one of the following two cases holds:

1. either $\theta_{\max }=\theta_{i}^{P}$ for some $i \in\left\{1, \ldots, n^{P}\right\}$ or

2. $\theta_{\max }=\theta_{i}^{C}$ for some $i \in\left\{1, \ldots, n^{C}\right\}$.

In the second case $\theta(x)=\theta_{\max }$ on $\Gamma_{i}^{C}$ or otherwise we would have

$$
0=\int_{\Gamma_{i}^{C}} k \frac{\partial \theta}{\partial \mathbf{n}} \mathrm{d} \Gamma=\int_{\Gamma_{i}^{C}} \alpha_{i}\left(\theta-\theta_{i}^{C}\right) \mathrm{d} \Gamma,
$$

and since $\alpha_{i}(x)>0$ and $\theta(x) \leqslant \theta_{i}^{C}=\theta_{\max } \forall x \in \bar{\Omega}$ this can only be achieved if $\theta=\theta_{i}^{C}$ on $\Gamma_{i}^{C}$.

Similar results can be obtained for the minimum.

Summarizing, temperature $\theta$ has to be constant on the boundary where $\theta_{\min }$ (respectively, $\theta_{\max }$ ) is attained and its value there has to be $\theta_{\min }$ (respectively, $\theta_{\max }$ ). Thus, we get a contradiction.

Moreover, we have

$$
\begin{aligned}
& \mathbf{K}\left(\theta_{1}^{P}, \ldots, \theta_{n^{P}}^{P}, \theta_{1}^{C}, \ldots, \theta_{n^{C}}^{C}\right) \cdot\left(\theta_{1}^{P}, \ldots, \theta_{n^{P}}^{P}, \theta_{1}^{C}, \ldots, \theta_{n^{C}}^{C}\right)=\sum_{i=1}^{n^{P}} \theta_{i}^{P} Q_{i}^{P}+\sum_{i=1}^{n^{C}} \theta_{i}^{C} Q_{i}^{C} \\
& =\int_{\Gamma^{P}} \theta k \frac{\partial \theta}{\partial \mathbf{n}} \mathrm{d} \Gamma+\sum_{i=1}^{n^{C}} \int_{\Gamma_{i}^{C}}\left(\frac{1}{\alpha_{i}} k \frac{\partial \theta}{\partial \mathbf{n}}+\theta\right) k \frac{\partial \theta}{\partial \mathbf{n}} \mathrm{d} \Gamma=\sum_{i=1}^{n^{C}} \int_{\Gamma_{i}^{C}} \frac{1}{\alpha_{i}}\left|k \frac{\partial \theta}{\partial \mathbf{n}}\right|^{2} \mathrm{~d} \Gamma+\int_{\Gamma} k \frac{\partial \theta}{\partial \mathbf{n}} \theta \mathrm{d} \Gamma \\
& =\sum_{i=1}^{n^{C}} \int_{\Gamma_{i}^{C}} \frac{1}{\alpha_{i}}\left|k \frac{\partial \theta}{\partial \mathbf{n}}\right|^{2} \mathrm{~d} \Gamma \int_{\Omega} k|\operatorname{grad} \theta|^{2} \mathrm{~d} x-\int_{\Omega} \operatorname{div}(k \operatorname{grad} \theta) \theta \mathrm{d} x \\
& =\sum_{i=1}^{n^{C}} \int_{\Gamma_{i}^{C}} \frac{1}{\alpha_{i}}\left|k \frac{\partial \theta}{\partial \mathbf{n}}\right|^{2} \mathrm{~d} \Gamma+\int_{\Omega} k|\operatorname{grad} \theta|^{2} \mathrm{~d} x \geqslant 0,
\end{aligned}
$$

where we have used a Green's formula and Equation (10). We note that the equality to 0 only holds for constant $\theta$, hence $\mathbf{K}$ is positive definite on the orthogonal space to $\operatorname{span}(\mathbf{e})$, as claimed.

Now we can characterize the image of $\mathbf{K}$. Since $\mathbf{K}$ is symmetric we have

$$
\operatorname{Im}(\mathbf{K})=\operatorname{Ker}(\mathbf{K})^{\perp}=\left\{\left(Q_{1}^{P}, \ldots, Q_{n^{P}}^{P}, Q_{1}^{C}, \ldots, Q_{n^{C}}^{C}\right) \in \mathbb{R}^{n^{P}+n^{C}}: \sum_{i=1}^{n^{P}} Q_{i}^{P}+\sum_{i=1}^{n^{C}} Q_{i}^{C}=0\right\} .
$$

The restriction of $\mathbf{K}$ to the quotient space,

$$
\mathscr{W}:=\frac{\mathbb{R}^{n^{P}+n^{C}}}{\operatorname{Ker}(\mathbf{K})} \simeq \operatorname{Im}(\mathbf{K}) \simeq \mathbb{R}^{n^{P}+n^{C}-1},
$$

to be denoted by $\tilde{\mathbf{K}}$, is called thermal conductance of the thermal system. We note that $\tilde{\mathbf{K}}$ depends only on the geometry (domain $\Omega$, ports and convective boundaries), the thermal 
conductivity $k$ and the convective heat transfer coefficients $\alpha_{i}$. The inverse of $\tilde{\mathbf{K}}$ is called thermal resistance and denoted by $\tilde{\mathbf{R}}$. We can choose the representative vector of each element in the quotient space as the one having its last component null. Then $\tilde{\mathbf{K}}$ can be identified to the isomorphism

$$
\tilde{\mathbf{K}}: \mathbb{R}^{n^{P}+n^{C}-1} \longrightarrow \mathbb{R}^{n^{P}+n^{C}-1}
$$

defined by

$$
\tilde{\mathbf{K}}\left(\theta_{1}^{P}, \ldots, \theta_{n^{P}}^{P}, \theta_{1}^{C}, \ldots, \theta_{n^{C}-1}^{C}\right)=\left(Q_{1}^{P}, \ldots, Q_{n^{P}}^{P}, Q_{1}^{C}, \ldots, Q_{n^{C}-1}^{C}\right) .
$$

We note that in the MKS system the unit for the entries of $[\tilde{\mathbf{R}}]$ is Kelvin per Watt $(K / W)$. An example is given below (see Example 1).

\section{THE ADAPTED REDUCED BASIS}

In order to solve problem $\mathbf{P}$ by a lumped parameter method we introduce an adapted reduced basis consisting of the temperature fields associated with the canonical basis of $\mathbb{R}^{n^{P}+n^{C}}$ through problem SP. More precisely, this basis is the set of time-independent functions $\left\{\varphi_{i}^{P}, i=1, \ldots, n^{P}\right\} \cup$ $\left\{\varphi_{i}^{C}, i=1, \ldots, n^{C}\right\}$ defined as the unique solutions to the following stationary boundary-value problems:

- For $j=1, \ldots, n^{P}$ find $\varphi_{j}^{P} \in H^{1}(\Omega)$ satisfying,

$$
\begin{aligned}
-\operatorname{div}\left(k \operatorname{grad} \varphi_{j}^{P}\right) & =0 \quad \text { in } \Omega, \\
\varphi_{j}^{P} & =\delta_{i j} \text { on } \Gamma_{i}^{P}, \quad i=1, \ldots, n^{P}, \\
k \frac{\partial \varphi_{j}^{P}}{\partial \mathbf{n}}+\alpha_{i} \varphi_{j}^{P} & =0 \quad \text { on } \Gamma_{i}^{C}, \quad i=1, \ldots, n^{C}, \\
k \frac{\partial \varphi_{j}^{P}}{\partial \mathbf{n}} & =0 \text { on } \Gamma^{A} .
\end{aligned}
$$

- For $j=1, \ldots n^{C}$ find $\varphi_{j}^{C} \in H^{1}(\Omega)$ satisfying,

$$
\begin{aligned}
-\operatorname{div}\left(k \operatorname{grad} \varphi_{j}^{C}\right)=0 & \text { in } \Omega, \\
\varphi_{j}^{C}=0 & \text { on } \Gamma_{i}^{P}, \quad i=1, \ldots, n^{P}, \\
k \frac{\partial \varphi_{j}^{C}}{\partial \mathbf{n}}+\alpha_{i}\left(\varphi_{j}^{C}-\delta_{i j}\right)=0 & \text { on } \Gamma_{i}^{C}, \quad i=1, \ldots, n^{C}, \\
k \frac{\partial \varphi_{j}^{C}}{\partial \mathbf{n}}=0 & \text { on } \Gamma^{A} .
\end{aligned}
$$

Their respective weak formulations are

$$
\begin{array}{r}
\int_{\Omega} k \operatorname{grad} \varphi_{j}^{P} \cdot \operatorname{grad} \psi \mathrm{d} x+\sum_{l=1}^{n^{C}} \int_{\Gamma_{l}^{C}} \alpha_{l} \varphi_{j}^{P} \psi \mathrm{d} \Gamma=0 \text { for all } \psi \in H^{1}(\Omega) \quad \text { null on } \Gamma^{P}, \\
\varphi_{j}^{P}=\delta_{i j} \quad \text { on } \Gamma_{i}^{P}, \quad i=1, \ldots, n^{P}, \quad j=1, \ldots, n^{P},
\end{array}
$$


and

$$
\int_{\Omega} k \operatorname{grad} \varphi_{j}^{C} \cdot \operatorname{grad} \psi \mathrm{d} x+\sum_{l=1}^{n^{C}} \int_{\Gamma_{l}^{C}} \alpha_{l} \varphi_{j}^{C} \psi \mathrm{d} \Gamma=0 \int_{\Gamma_{j}^{C}} \alpha_{j} \psi \mathrm{d} \Gamma
$$

for all $\psi \in H^{1}(\Omega)$ null on $\Gamma^{P}$,

$$
\varphi_{j}^{C}=0 \quad \text { on } \Gamma_{i}^{P}, i=1, \ldots, n^{P}, j=1, \ldots, n^{C} .
$$

Since we have assumed that ports are pairwise disjoint, we can find functions in $H^{1}(\Omega)$ satisfying boundary conditions (27). Then, by using the Lax-Milgram Lemma, it is straightforward to prove that the above problems have unique solutions.

In general, these weak formulations are solved by using finite element methods. We emphasize that they are time independent and that the stiffness matrix is the same for all the $n^{P}+n^{C}$ problems. Moreover, since this matrix is symmetric and positive definite it can be assembled and factorized only once. Thus, computing functions $\varphi_{j}^{P}$ and $\varphi_{j}^{C}$ is not very costly.

Let

$$
\varphi(x):=\sum_{i=1}^{n^{P}} b_{i}^{P} \varphi_{i}^{P}(x)+\sum_{i=1}^{n^{C}} b_{i}^{C} \varphi_{i}^{C}(x) .
$$

Then it is straightforward to see that

$$
\begin{aligned}
\varphi(x) & =b_{i}^{P} \forall x \in \Gamma_{i}^{P}, \quad i=1, \ldots, n^{P}, \\
\varphi(x)+\frac{k}{\alpha_{i}} \frac{\partial \varphi}{\partial \mathbf{n}}(x) & =b_{i}^{C} \forall x \in \Gamma_{i}^{C}, \quad i=1, \ldots, n^{C} .
\end{aligned}
$$

As a consequence, the $n^{P}+n^{C}$ above functions are linearly independent. Moreover,

$$
\sum_{i=1}^{n^{P}} \varphi_{i}^{P}(x)+\sum_{i=1}^{n^{C}} \varphi_{i}^{C}(x)=1 \forall x \in \Omega
$$

According to the discussion in Section 2, we observe that for $j=1, \ldots, n^{P}$ the $j$ th column of matrix $[\mathbf{K}]$ is the vector given by

$$
\left(\int_{\Gamma_{1}^{P}} k \frac{\partial \varphi_{j}^{P}}{\partial \mathbf{n}} \mathrm{d} \Gamma, \ldots, \int_{\Gamma_{n}^{P}} k \frac{\partial \varphi_{j}^{P}}{\partial \mathbf{n}} \mathrm{d} \Gamma, \int_{\Gamma_{1}^{C}} k \frac{\partial \varphi_{j}^{P}}{\partial \mathbf{n}} \mathrm{d} \Gamma, \ldots, \int_{\Gamma_{n}^{C} C} k \frac{\partial \varphi_{j}^{P}}{\partial \mathbf{n}} \mathrm{d} \Gamma\right)^{\mathrm{T}}
$$

Similarly, for $j=1, \ldots, n^{C}$, the $\left(j+n^{P}\right)$ th column is

$$
\left(\int_{\Gamma_{1}^{P}} k \frac{\partial \varphi_{j}^{C}}{\partial \mathbf{n}} \mathrm{d} \Gamma, \ldots, \int_{\Gamma_{n}^{P}} k \frac{\partial \varphi_{j}^{C}}{\partial \mathbf{n}} \mathrm{d} \Gamma, \int_{\Gamma_{1}^{C}} k \frac{\partial \varphi_{j}^{C}}{\partial \mathbf{n}} \mathrm{d} \Gamma, \ldots, \int_{\Gamma_{n}^{C}} k \frac{\partial \varphi_{j}^{C}}{\partial \mathbf{n}} \mathrm{d} \Gamma\right)^{\mathrm{T}} .
$$

Note that function $\varphi \equiv 1$ is the solution to problem $\mathbf{S P}$ for $\left(\theta_{1}^{P}, \ldots, \theta_{n^{P}}^{P}, \theta_{1}^{C}, \ldots, \theta_{n^{C}}^{C}\right)=\mathbf{e}$, which belongs to the kernel of $\mathbf{K}$. This implies that conductance matrix $[\tilde{\mathbf{K}}]$ can be obtained from the latter by deleting any row and the same column, for instance, the last ones.

\section{Example 1}

Let us assume that domain $\Omega$ is a cylinder of length $L$; more precisely $\Omega=\omega \times[0, L]$. Let us take

$$
\Gamma_{1}^{P}=\omega \times\{0\}, \Gamma_{2}^{P}=\omega \times\{L\} \quad \text { and } \quad \Gamma^{A}=\partial \omega \times[0, L] .
$$

Then $n^{P}=2, n^{C}=0$ and the adapted reduced basis consists of the two functions

$$
\varphi_{1}^{P}(x)=1-\frac{x_{3}}{L}, \quad \varphi_{2}^{P}(x)=\frac{x_{3}}{L} .
$$


Therefore,

$$
[\mathbf{K}]=\left(\begin{array}{cc}
\frac{k \operatorname{meas}(\omega)}{L} & -\frac{k \operatorname{meas}(\omega)}{L} \\
-\frac{k \operatorname{meas}(\omega)}{L} & \frac{k \operatorname{meas}(\omega)}{L}
\end{array}\right),
$$

where meas $(\omega)$ denotes the area of $\omega$. This matrix is clearly singular. Then the conductance matrix is

$$
[\tilde{\mathbf{K}}]=\left(\frac{k \operatorname{meas}(\omega)}{L}\right)
$$

and the resistance matrix is

$$
[\mathbf{R}]=\left(\frac{L}{k \operatorname{meas}(\omega)}\right)
$$

It is worth pointing out that the latter is the formula for the electric resistance of a cylindrical conductor with electric conductivity $k$.

The following alternative expressions for the entries of matrix $[\mathbf{K}]$ are useful from the computational point of view. Let us write this matrix in block form, namely,

$$
[\mathbf{K}]=\left(\begin{array}{ll}
K^{P P} & K^{P C} \\
K^{C P} & K^{C C}
\end{array}\right)
$$

\section{Proposition 2}

The following equalities hold:

1. $K_{i j}^{P P}:=\int_{\Gamma_{i}^{P}} k \frac{\partial \varphi_{j}^{P}}{\partial \mathbf{n}} \mathrm{d} \Gamma=\int_{\Omega} k \operatorname{grad} \varphi_{j}^{P} \cdot \operatorname{grad} \varphi_{i}^{P} \mathrm{~d} x+\sum_{l=1}^{n^{C}} \int_{\Gamma_{l}^{C}} \alpha_{l} \varphi_{j}^{P} \varphi_{i}^{P} \mathrm{~d} \Gamma, \quad i, j=1, \ldots, n^{P}$,

2.

$$
K_{i j}^{P C}:=\int_{\Gamma_{i}^{C}} k \frac{\partial \varphi_{j}^{P}}{\partial \mathbf{n}} \mathrm{d} \Gamma=-\int_{\Gamma_{i}^{C}} \alpha_{i} \varphi_{j}^{P} \mathrm{~d} \Gamma, \quad i=1, \ldots, n^{C}, j=1, \ldots, n^{P},
$$

3.

$$
K_{i j}^{C P}:=\int_{\Gamma_{i}^{P}} k \frac{\partial \varphi_{j}^{C}}{\partial \mathbf{n}} \mathrm{d} \Gamma=-\int_{\Gamma_{j}^{C}} \alpha_{j} \varphi_{i}^{P} \mathrm{~d} \Gamma, \quad i=1, \ldots, n^{P}, j=1, \ldots, n^{C},
$$

4.

$$
K_{i j}^{C C}:=\int_{\Gamma_{i}^{C}} k \frac{\partial \varphi_{j}^{C}}{\partial \mathbf{n}} \mathrm{d} \Gamma=\delta_{i j} \int_{\Gamma_{i}^{C}} \alpha_{i} \mathrm{~d} \Gamma-\int_{\Gamma_{i}^{C}} \alpha_{i} \varphi_{j}^{C} \mathrm{~d} \Gamma, \quad i, j=1, \ldots, n^{C} .
$$

Proof

We have

1.

$$
\begin{aligned}
\int_{\Gamma_{i}^{P}} k \frac{\partial \varphi_{j}^{P}}{\partial \mathbf{n}} \mathrm{d} \Gamma & =\sum_{l=1}^{n^{P}} \int_{\Gamma_{l}^{P}} k \frac{\partial \varphi_{j}^{P}}{\partial \mathbf{n}} \varphi_{i}^{P} \mathrm{~d} \Gamma=\int_{\Omega} k \operatorname{grad} \varphi_{j}^{P} \cdot \operatorname{grad} \varphi_{i}^{P} \mathrm{~d} x-\sum_{l=1}^{n^{C}} \int_{\Gamma_{l}^{C}} k \frac{\partial \varphi_{j}^{P}}{\partial \mathbf{n}} \varphi_{i}^{P} \mathrm{~d} \Gamma \\
& =\int_{\Omega} k \operatorname{grad} \varphi_{j}^{P} \cdot \operatorname{grad} \varphi_{i}^{P} \mathrm{~d} x+\sum_{l=1}^{n^{C}} \int_{\Gamma_{l}^{C}} \alpha_{l} \varphi_{j}^{P} \varphi_{i}^{P} \mathrm{~d} \Gamma
\end{aligned}
$$

by using (19) and (20). 
2.

by using (20).

$$
\int_{\Gamma_{i}^{C}} k \frac{\partial \varphi_{j}^{P}}{\partial \mathbf{n}} \mathrm{d} \Gamma=-\int_{\Gamma_{i}^{C}} \alpha_{i} \varphi_{j}^{P} \mathrm{~d} \Gamma
$$

3. First we have

$$
\int_{\Omega} k \operatorname{grad} \varphi_{j}^{C} \cdot \operatorname{grad} \varphi_{i}^{P} \mathrm{~d} x+\sum_{l=1}^{n^{C}} \int_{\Gamma_{l}^{C}} \alpha_{l} \varphi_{j}^{C} \varphi_{i}^{P} \mathrm{~d} \Gamma=0 .
$$

Indeed

$$
\begin{aligned}
& \int_{\Omega} k \operatorname{grad} \varphi_{j}^{C} \cdot \operatorname{grad} \varphi_{i}^{P} \mathrm{~d} x+\sum_{l=1}^{n^{C}} \int_{\Gamma_{l}^{C}} \alpha_{l} \varphi_{j}^{C} \varphi_{i}^{P} \mathrm{~d} \Gamma \\
& =\sum_{l=1}^{n^{P}} \int_{\Gamma_{l}^{P}} k \frac{\partial \varphi_{i}^{P}}{\partial \mathbf{n}} \varphi_{j}^{C} \mathrm{~d} \Gamma+\sum_{l=1}^{n^{C}} \int_{\Gamma_{l}^{C}} k \frac{\partial \varphi_{i}^{P}}{\partial \mathbf{n}} \varphi_{j}^{C} \mathrm{~d} \Gamma \\
& \quad+\sum_{l=1}^{n^{C}} \int_{\Gamma_{l}^{C}} \alpha_{l} \varphi_{j}^{C} \varphi_{i}^{P} \mathrm{~d} \Gamma=\sum_{l=1}^{n^{C}} \int_{\Gamma_{l}^{C}}\left(k \frac{\partial \varphi_{i}^{P}}{\partial \mathbf{n}}+\alpha_{l} \varphi_{i}^{P}\right) \varphi_{j}^{C} \mathrm{~d} \Gamma=0
\end{aligned}
$$

by using (23) and (20).

Moreover,

$$
\begin{aligned}
\int_{\Gamma_{i}^{P}} k \frac{\partial \varphi_{j}^{C}}{\partial \mathbf{n}} \mathrm{d} \Gamma & =\sum_{l=1}^{n^{P}} \int_{\Gamma_{l}^{P}} k \frac{\partial \varphi_{j}^{C}}{\partial \mathbf{n}} \varphi_{i}^{P} \mathrm{~d} \Gamma=\int_{\Omega} k \operatorname{grad} \varphi_{j}^{C} \cdot \operatorname{grad} \varphi_{i}^{P} \mathrm{~d} x-\sum_{l=1}^{n^{C}} \int_{\Gamma_{l}^{C}} k \frac{\partial \varphi_{j}^{C}}{\partial \mathbf{n}} \varphi_{i}^{P} \mathrm{~d} \Gamma \\
& =\int_{\Omega} k \operatorname{grad} \varphi_{j}^{C} \cdot \operatorname{grad} \varphi_{i}^{P} \mathrm{~d} x-\sum_{l=1}^{n^{C}} \int_{\Gamma_{l}^{C}} \alpha_{l}\left(\delta_{l j}-\varphi_{j}^{C}\right) \varphi_{i}^{P} \mathrm{~d} \Gamma \\
& =\int_{\Omega} k \operatorname{grad} \varphi_{j}^{C} \cdot \operatorname{grad} \varphi_{i}^{P} \mathrm{~d} x+\sum_{l=1}^{n^{C}} \int_{\Gamma_{l}^{C}} \alpha_{l} \varphi_{j}^{C} \varphi_{i}^{P} \mathrm{~d} \Gamma-\int_{\Gamma_{j}^{C}} \alpha_{j} \varphi_{i}^{P} \mathrm{~d} \Gamma \\
& =-\int_{\Gamma_{j}^{C}} \alpha_{j} \varphi_{i}^{P} \mathrm{~d} \Gamma .
\end{aligned}
$$

4. Finally

$$
\int_{\Gamma_{i}^{C}} k \frac{\partial \varphi_{j}^{C}}{\partial \mathbf{n}} \mathrm{d} \Gamma=\int_{\Gamma_{i}^{C}} \alpha_{i}\left(\delta_{i j}-\varphi_{j}^{C}\right) \mathrm{d} \Gamma=\delta_{i j} \int_{\Gamma_{i}^{C}} \alpha_{i} \mathrm{~d} \Gamma-\int_{\Gamma_{i}^{C}} \alpha_{i} \varphi_{j}^{C} \mathrm{~d} \Gamma
$$

from (24).

Corollary 3

Matrix $[\mathbf{K}]$ is symmetric.

Proof

According to the previous computations we only need to show that $K_{i j}^{C C}=K_{j i}^{C C}$ or, equivalently, that

$$
\int_{\Gamma_{i}^{C}} \alpha_{i} \varphi_{j}^{C} \mathrm{~d} \Gamma=\int_{\Gamma_{j}^{C}} \alpha_{j} \varphi_{i}^{C} \mathrm{~d} \Gamma
$$


For this purpose we note that

$$
\begin{aligned}
\int_{\Gamma_{i}^{C}} \alpha_{i} \varphi_{j}^{C} \mathrm{~d} \Gamma & =\sum_{l=1}^{n^{C}} \int_{\Gamma_{l}^{C}} k \frac{\partial \varphi_{i}^{C}}{\partial \mathbf{n}} \varphi_{j}^{C} \mathrm{~d} \Gamma+\sum_{l=1}^{n^{C}} \int_{\Gamma_{l}^{C}} \alpha_{l} \varphi_{i}^{C} \varphi_{j}^{C} \mathrm{~d} \Gamma \\
& =\sum_{l=1}^{n^{P}} \int_{\Gamma_{l}^{P}} k \frac{\partial \varphi_{i}^{C}}{\partial \mathbf{n}} \varphi_{j}^{C} \mathrm{~d} \Gamma+\sum_{l=1}^{n^{C}} \int_{\Gamma_{l}^{C}} k \frac{\partial \varphi_{i}^{C}}{\partial \mathbf{n}} \varphi_{j}^{C} \mathrm{~d} \Gamma+\sum_{l=1}^{n^{C}} \int_{\Gamma_{l}^{C}} \alpha_{l} \varphi_{i}^{C} \varphi_{j}^{C} \mathrm{~d} \Gamma \\
& =\int_{\Omega} k \operatorname{grad} \varphi_{i}^{C} \cdot \operatorname{grad} \varphi_{j}^{C} \mathrm{~d} x+\sum_{l=1}^{n^{C}} \int_{\Gamma_{l}^{C}} \alpha_{l} \varphi_{i}^{C} \varphi_{j}^{C} \mathrm{~d} \Gamma
\end{aligned}
$$

Now the result follows because the last expression is symmetric with respect to $i$ and $j$.

\section{THE TRANSIENT PROBLEM. THERMAL CAPACITANCE}

Let us come back to the transient problem (1)-(5). We seek a solution that, at each time $t$, belongs to the space $\mathscr{Z}$ spanned by the (time independent) reduced basis introduced in the previous section. More precisely, we look for an approximate solution of the form

$$
\hat{\theta}(x, t):=\sum_{j=1}^{n^{P}} \theta_{j}^{P}(t) \varphi_{j}^{P}(x)+\sum_{j=1}^{n^{C}} \theta_{j}^{C}(t) \varphi_{j}^{C}(x) .
$$

In order to determine the time-dependent coefficients $\theta_{i}^{P}, i=1, \ldots, n^{P}$ and $\theta_{i}^{C}, i=1, \ldots, n^{C}$, we introduce the following semi-discrete problem:

$$
\begin{aligned}
& \sum_{j=1}^{n^{P}} \frac{\mathrm{d} \theta_{j}^{P}}{\mathrm{~d} t}(t) \int_{\Omega} \rho c \varphi_{j}^{P} \psi \mathrm{d} x+\sum_{j=1}^{n^{C}} \frac{\mathrm{d} \theta_{j}^{C}}{\mathrm{~d} t}(t) \int_{\Omega} \rho c \varphi_{j}^{C} \psi \mathrm{d} x+\sum_{j=1}^{n^{P}} \theta_{j}^{P}(t) \int_{\Omega} k \operatorname{grad} \varphi_{j}^{P} \cdot \operatorname{grad} \psi \mathrm{d} x \\
& \quad+\sum_{j=1}^{n^{C}} \theta_{j}^{C}(t) \int_{\Omega} k \operatorname{grad} \varphi_{j}^{C} \cdot \operatorname{grad} \psi \mathrm{d} x+\sum_{j=1}^{n^{P}} \theta_{j}^{P}(t) \sum_{l=1}^{n^{C}} \int_{\Gamma_{l}^{C}} \alpha_{l} \varphi_{j}^{P} \psi \mathrm{d} \Gamma \\
& \quad+\sum_{j=1}^{n^{C}} \theta_{j}^{C}(t) \sum_{l=1}^{n^{C}} \int_{\Gamma_{l}^{C}} \alpha_{l} \varphi_{j}^{C} \psi \mathrm{d} \Gamma \\
& =\int_{\Omega} f \psi \mathrm{d} x+\sum_{l=1}^{n^{C}} \int_{\Gamma_{l}^{C}} \alpha_{l} \tilde{\theta}_{l}^{C} \psi \mathrm{d} \Gamma \text { for all } \psi \in \mathscr{Z} .
\end{aligned}
$$

Equality (33) shows that the constant function $\psi \equiv 1$ belongs to the space of test functions. This fact allows us to prove the following result.

Lemma 4

The solution of the above semi-discrete problem satisfies the following energy conservation principle:

$$
\frac{\mathrm{d}}{\mathrm{d} t} \int_{\Omega} \rho c \hat{\theta}(x, t) \mathrm{d} x=\int_{\Omega} f \mathrm{~d} x+\sum_{l=1}^{n^{C}} \int_{\Gamma_{l}^{C}} \alpha_{l}\left(\tilde{\theta}_{l}^{C}-\hat{\theta}(x, t)\right) \mathrm{d} \Gamma .
$$

Moreover, by replacing the test function $\psi$ by the basis elements $\varphi_{i}^{P}$ and $\varphi_{i}^{C}$ we see that problem (35) is equivalent to an ordinary differential equation system which can be written in a compact way as follows:

$$
M \frac{\mathrm{d} \Theta}{\mathrm{d} t}+A \Theta=\mathbf{b}
$$


where

$$
\boldsymbol{\Theta}(t)=\left(\theta_{1}^{P}(t), \ldots, \theta_{n^{P}}^{P}(t), \theta_{1}^{C}(t), \ldots, \theta_{n^{C}}^{C}(t)\right)^{\mathrm{T}},
$$

$M$ is the so-called thermal capacitance matrix given by

$$
M=\left(\begin{array}{ll}
M^{P P} & M^{P C} \\
M^{C P} & M^{C C}
\end{array}\right)
$$

with

$$
\begin{aligned}
M_{i j}^{P P} & =\int_{\Omega} \rho c \varphi_{i}^{P} \varphi_{j}^{P} \mathrm{~d} x, i, j=1, \ldots, n^{P}, \\
M_{i j}^{P C} & =\int_{\Omega} \rho c \varphi_{j}^{C} \varphi_{i}^{P} \mathrm{~d} x, i=1, \ldots, n^{P}, j=1, \ldots, n^{C}, \\
M_{i j}^{C P} & =\int_{\Omega} \rho c \varphi_{j}^{P} \varphi_{i}^{C} \mathrm{~d} x, i=1, \ldots, n^{C}, j=1, \ldots, n^{P}, \\
M_{i j}^{C C} & =\int_{\Omega} \rho c \varphi_{i}^{C} \varphi_{j}^{C} \mathrm{~d} x, i, j=1, \ldots, n^{C} .
\end{aligned}
$$

Similarly, the 'stiffness' matrix $A$ is given by

$$
\begin{gathered}
A=\left(\begin{array}{cc}
A^{P P} & A^{P C} \\
A^{C P} & A^{C C}
\end{array}\right) \\
A_{i j}^{P P}=\int_{\Omega} k \operatorname{grad} \varphi_{j}^{P} \cdot \operatorname{grad} \varphi_{i}^{P} \mathrm{~d} x+\sum_{l=1}^{n^{C}} \int_{\Gamma_{l}^{C}} \alpha_{l} \varphi_{j}^{P} \varphi_{i}^{P} \mathrm{~d} \Gamma, \quad i, j=1, \ldots n^{P}, \\
A_{i j}^{P C}=\int_{\Omega} k \operatorname{grad} \varphi_{j}^{C} \cdot \operatorname{grad} \varphi_{i}^{P} \mathrm{~d} x+\sum_{l=1}^{n^{C}} \int_{\Gamma_{l}^{C}} \alpha_{l} \varphi_{j}^{C} \varphi_{i}^{P} \mathrm{~d} \Gamma, \quad i=1, \ldots, n^{C}, \quad j=1, \ldots, n^{P}, \\
A_{i j}^{C P}=\int_{\Omega} k \operatorname{grad} \varphi_{j}^{P} \cdot \operatorname{grad} \varphi_{i}^{C} \mathrm{~d} x+\sum_{l=1}^{n^{C}} \int_{\Gamma_{l}^{C}} \alpha_{l} \varphi_{j}^{P} \varphi_{i}^{C} \mathrm{~d} \Gamma, \quad i=1, \ldots, n^{C}, \quad j=1, \ldots, n^{P}, \\
A_{i j}^{C C}=\int_{\Omega} k \operatorname{grad} \varphi_{j}^{C} \cdot \operatorname{grad} \varphi_{i}^{C} \mathrm{~d} x+\sum_{l=1}^{n^{C}} \int_{\Gamma_{l}^{C}} \alpha_{l} \varphi_{j}^{C} \varphi_{i}^{C} \mathrm{~d} \Gamma, \quad i, j=1, \ldots, n^{C} .
\end{gathered}
$$

Finally, vector $\mathbf{b}$ is defined by

$$
\mathbf{b}=\left(\begin{array}{l}
b^{P} \\
b^{C}
\end{array}\right),
$$

with

$$
\begin{aligned}
& b_{i}^{P}(t)=\int_{\Omega} f \varphi_{i}^{P} \mathrm{~d} x+\sum_{l=1}^{n^{C}} \int_{\Gamma_{l}^{C}} \alpha_{l} \tilde{\theta}_{l}^{C} \varphi_{i}^{P} \mathrm{~d} \Gamma, \quad i=1, \ldots, n^{P}, \\
& b_{i}^{C}(t)=\int_{\Omega} f \varphi_{i}^{C} \mathrm{~d} x+\sum_{l=1}^{n^{C}} \int_{\Gamma_{l}^{C}} \alpha_{l} \tilde{\theta}_{l}^{C} \varphi_{i}^{C} \mathrm{~d} \Gamma, \quad i=1, \ldots, n^{C} .
\end{aligned}
$$


Now, let us explore the relation between matrix A and matrix $[\mathbf{K}]$. We have

$$
\begin{aligned}
A_{i j}^{P P}= & \int_{\Omega} k \operatorname{grad} \varphi_{j}^{P} \cdot \operatorname{grad} \varphi_{i}^{P} \mathrm{~d} x+\sum_{l=1}^{n^{C}} \int_{\Gamma_{l}^{C}} \alpha_{l} \varphi_{j}^{P} \varphi_{i}^{P} \mathrm{~d} \Gamma=\sum_{l=1}^{n^{P}} \int_{\Gamma_{l}^{P}} k \frac{\partial \varphi_{j}^{P}}{\partial \mathbf{n}} \varphi_{i}^{P} \mathrm{~d} \Gamma \\
& +\sum_{l=1}^{n^{C}} \int_{\Gamma_{l}^{C}} k \frac{\partial \varphi_{j}^{P}}{\partial \mathbf{n}} \varphi_{i}^{P} \mathrm{~d} \Gamma+\sum_{l=1}^{n^{C}} \int_{\Gamma_{l}^{C}} \alpha_{l} \varphi_{j}^{P} \varphi_{i}^{P} \mathrm{~d} \Gamma=\int_{\Gamma_{i}^{P}} k \frac{\partial \varphi_{j}^{P}}{\partial \mathbf{n}} \mathrm{d} \Gamma=K_{i j}^{P P}
\end{aligned}
$$

from (19) and (20).

$$
\begin{aligned}
A_{i j}^{P C} & =A_{j i}^{C P}=\int_{\Omega} k \operatorname{grad} \varphi_{j}^{P} \cdot \operatorname{grad} \varphi_{i}^{C} \mathrm{~d} x+\sum_{l=1}^{n^{C}} \int_{\Gamma_{l}^{C}} \alpha_{l} \varphi_{j}^{P} \varphi_{i}^{C} \mathrm{~d} \Gamma \\
& =\sum_{l=1}^{n^{P}} \int_{\Gamma_{l}^{P}} k \frac{\partial \varphi_{j}^{P}}{\partial \mathbf{n}} \varphi_{i}^{C} \mathrm{~d} \Gamma+\sum_{l=1}^{n^{C}} \int_{\Gamma_{l}^{C}} k \frac{\partial \varphi_{j}^{P}}{\partial \mathbf{n}} \varphi_{i}^{C} \mathrm{~d} \Gamma+\sum_{l=1}^{n^{C}} \int_{\Gamma_{l}^{C}} \alpha_{l} \varphi_{j}^{P} \varphi_{i}^{C} \mathrm{~d} \Gamma \\
& =K_{i j}^{P C}+\int_{\Gamma_{i}^{C}} \alpha_{i} \varphi_{j}^{P} \mathrm{~d} \Gamma
\end{aligned}
$$

from (23) and (20).

$$
\begin{aligned}
A_{i j}^{C C}= & \int_{\Omega} k \operatorname{grad} \varphi_{j}^{C} \cdot \operatorname{grad} \varphi_{i}^{C} \mathrm{~d} x+\sum_{l=1}^{n^{C}} \int_{\Gamma_{l}^{C}} \alpha_{l} \varphi_{j}^{C} \varphi_{i}^{C} \mathrm{~d} \Gamma=\sum_{l=1}^{n^{P}} \int_{\Gamma_{l}^{P}} k \frac{\partial \varphi_{j}^{C}}{\partial \mathbf{n}} \varphi_{i}^{C} \mathrm{~d} \Gamma \\
& +\sum_{l=1}^{n^{C}} \int_{\Gamma_{l}^{C}} k \frac{\partial \varphi_{j}^{C}}{\partial \mathbf{n}} \varphi_{i}^{C} \mathrm{~d} \Gamma+\sum_{l=1}^{n^{C}} \int_{\Gamma_{l}^{C}} \alpha_{l} \varphi_{j}^{C} \varphi_{i}^{C} \mathrm{~d} \Gamma \\
= & \int_{\Gamma_{j}^{C}} \alpha_{j} \varphi_{i}^{C} \mathrm{~d} \Gamma=\delta_{i j} \int_{\Gamma_{i}^{C}} \alpha_{i} \mathrm{~d} \Gamma-K_{i j}^{C C} .
\end{aligned}
$$

Remark 5

We note that the capacitance matrix $M$ is symmetric and positive definite. Thus, initial-value problems for the ordinary differential equation system (37) are well posed.

\section{Example 6}

In the case of the geometry of Example 1, assuming that all thermo-physical parameters are constant in $\Omega$ we have

$$
\begin{aligned}
& A_{11}=\frac{k \operatorname{meas}(\omega)}{L}, \\
& A_{12}=A_{21}=-\frac{k \operatorname{meas}(\omega)}{L}, \\
& A_{22}=\frac{k \operatorname{meas}(\omega)}{L},
\end{aligned}
$$

and

$$
\begin{aligned}
& M_{11}=M_{22}=\rho c \int_{\Omega}\left(1-\frac{x_{3}}{L}\right)^{2} \mathrm{~d} x=\frac{1}{3} \rho c \operatorname{meas}(\omega) L=\frac{1}{3} \rho c \operatorname{meas}(\Omega), \\
& M_{12}=M_{21}=\rho c \int_{\Omega}\left(1-\frac{x_{3}}{L}\right) \frac{x_{3}}{L} \mathrm{~d} x=\frac{1}{6} \rho c \operatorname{meas}(\omega) L=\frac{1}{6} \rho c \operatorname{meas}(\Omega) .
\end{aligned}
$$




\section{LUMPED PARAMETER DOMAIN DECOMPOSITION METHODS}

In this section we combine the above ideas to introduce lumped parameter models with domain decomposition techniques (see, for instance, $[13,14]$ ). For this purpose we decompose domain $\Omega$ into subdomains $\Omega_{i}, i=1, \ldots, N$, connected among them through boundaries to be called 'ports'. Thus, in the boundary of each subdomain $\Omega_{i}$, called $\Gamma_{i}$, we distinguish three parts,

- the ports,

$$
\Gamma_{i}^{P}=\bigcup_{j=1}^{n_{i}^{P}} \Gamma_{i j}^{P} .
$$

- the convective boundary,

$$
\Gamma_{i}^{C}=\bigcup_{j=1}^{n_{i}^{C}} \Gamma_{i j}^{C},
$$

- the adiabatic boundary, $\Gamma_{i}^{A}$.

We assume that each $\Gamma_{i}^{P}, i=1, \ldots, n^{P}$ has been written as the union of its connected components.

In order to compute an approximate solution to problem (6), (7), we introduce an adapted reduced basis for the whole domain $\Omega$, by gluing the ones for each subdomain obtained in Section 4 . Recall that the basis for the $i$ th subdomain consists of $n_{i}^{P}+n_{i}^{C}$ elements, to be called $\varphi_{i j}^{P}: j=1, \ldots, n_{i}^{P}$, and $\varphi_{i j}^{C}: j=1, \ldots, n_{i}^{C}$ which are defined, respectively, as the unique solutions to the following boundary value problems:

- For $i=1, \ldots, N$ and $j=1, \ldots, n_{i}^{P}$ find $\varphi_{i j}^{P} \in H^{1}\left(\Omega_{i}\right)$ satisfying,

$$
\begin{aligned}
-\operatorname{div}\left(k \operatorname{grad} \varphi_{i j}^{P}\right) & =0 \quad \text { in } \Omega_{i}, \\
\varphi_{i j}^{P}(x) & =\delta_{j l} \text { on } \Gamma_{i l}^{P}, \quad l=1, \ldots, n_{i}^{P}, \\
k \frac{\partial \varphi_{i j}^{P}}{\partial \mathbf{n}}+\alpha_{l} \varphi_{i j}^{P} & =0 \text { on } \Gamma_{i l}^{C}, \quad l=1, \ldots, n_{i}^{C}, \\
k \frac{\partial \varphi_{i j}^{P}}{\partial \mathbf{n}} & =0 \text { on } \Gamma_{i}^{A} .
\end{aligned}
$$

- For $i=1, \ldots, N$ and $j=1, \cdots n_{i}^{C}$ find $\varphi_{i j}^{C} \in H^{1}\left(\Omega_{i}\right)$ satisfying,

$$
\begin{aligned}
-\operatorname{div}\left(k \operatorname{grad} \varphi_{i j}^{C}\right) & =0 \quad \text { in } \Omega, \\
\varphi_{i j}^{C}(x)=0 & \text { on } \Gamma_{i l}^{P}, \quad l=1, \ldots, n_{i}^{P}, \\
k \frac{\partial \varphi_{i j}^{C}}{\partial \mathbf{n}}+\alpha_{l}\left(\varphi_{i j}^{C}-\delta_{j l}\right)=0 & \text { on } \Gamma_{i l}^{C}, \quad l=1, \ldots, n_{i}^{C}, \\
k \frac{\partial \varphi_{i j}^{C}}{\partial \mathbf{n}} & =0 \text { on } \Gamma_{i}^{A} .
\end{aligned}
$$

With these functions it is straightforward to introduce an approximation space to functions defined in the whole domain $\Omega$. First, the ports are defined as the maximal elements of the set 
$\left\{\Gamma_{i l}^{P}: l=1, \ldots, n_{i}^{P}, i=1, \ldots, N\right\}$ with respect to the inclusion relation. Let us denote by $n^{P}$ the total number of ports and by $n^{C}$ the total number of convective boundaries. We note that

$$
n^{C}=\sum_{i=1}^{N} n_{i}^{C}
$$

but

$$
n^{P} \leqslant \frac{1}{2} \sum_{i=1}^{N} n_{i}^{P}
$$

because each port is shared by at least two subdomains.

Let us introduce a set of linear independent scalar fields, $\left\{w_{l}^{P}: l=1, \ldots, n^{P}\right\} \cup\left\{w_{l}^{C}: l=\right.$ $\left.1, \ldots, n^{C}\right\}$, defined in $\Omega$ as follows:

1. For the $l$ th global port, let $i_{k}, k=1, \ldots, m_{l}$, be the numbers of the $m_{l}$ subdomains sharing it and $j_{k}$ the corresponding number of this port in the local numbering of ports of domain $\Omega_{i_{k}}, k=1, \ldots, m_{l}$. Then $w_{l}^{P}: \Omega \longrightarrow \mathbb{R}$ is defined by

$$
\begin{aligned}
& w_{l}^{P}(x):=\varphi_{i_{k} j_{k}}^{P}(x) \text { in } \Omega_{i_{k}}, \\
& w_{l}^{P}(x):=0 \text { in } \Omega_{m} \text { for } m \neq i_{k}, k=1, \ldots, m_{l} .
\end{aligned}
$$

2. For the $l$ th global convective boundary, let $\Omega_{i}$ be the (unique) subdomain of which it is the convective boundary number $j$. Then $w_{l}^{C}: \Omega \longrightarrow \mathbb{R}$ is defined by

$$
\begin{aligned}
& w_{l}^{C}(x):=\varphi_{i j}^{C}(x) \text { in } \Omega_{i}, \\
& w_{l}^{C}(x):=0 \quad \text { in } \Omega_{m} \quad \text { for } m \neq i .
\end{aligned}
$$

Let us call $\mathscr{V}$ the linear space spanned by the above set of $n^{P}+n^{C}$ functions. The lumped parameter model is defined as the Galerkin approximation of problem $\mathbf{P}$ corresponding to this basis, namely,

LP. For $t \in[0, T]$, find $\tilde{\theta}(., t) \in \mathscr{V}$ satisfying

$$
\begin{aligned}
& \int_{\Omega} \rho c \frac{\partial \tilde{\theta}}{\partial t} \tilde{\psi} \mathrm{d} x+\int_{\Omega} k \operatorname{grad} \tilde{\theta} \cdot \operatorname{grad} \tilde{\psi} \mathrm{d} x+\sum_{l=1}^{n^{C}} \int_{\Gamma_{l}^{C}} \alpha_{l} \tilde{\theta} \tilde{\psi} \mathrm{d} \Gamma \\
& =\int_{\Omega} f \tilde{\psi} \mathrm{d} x+\sum_{l=1}^{n^{C}} \int_{\Gamma_{l}^{C}} \alpha_{l} \tilde{\theta}_{i}^{C} \tilde{\psi} \mathrm{d} \Gamma \quad \forall \tilde{\psi} \in \mathscr{V} \\
& \tilde{\theta}(x, 0)=\tilde{\theta}_{0}(x) \text { in } \Omega,
\end{aligned}
$$

where $\tilde{\theta}_{0}$ denotes a projection of the initial condition $\theta_{0}$ on the space $\mathscr{V}$.

By writing

$$
\tilde{\theta}(x, t)=\sum_{l=1}^{n^{P}} \theta_{l}^{P}(t) w_{l}^{P}(x)+\sum_{l=1}^{n^{C}} \theta_{l}^{C}(t) w_{l}^{C}(x),
$$

problem (68), (69) becomes an ordinary differential system of equations similar to (37), namely,

$$
\tilde{M} \frac{d \tilde{\mathbf{\Theta}}}{d t}+\tilde{A} \tilde{\mathbf{\Theta}}=\tilde{\mathbf{b}},
$$

where

$$
\tilde{\boldsymbol{\Theta}}(t)=\left(\theta_{1}^{P}(t), \ldots, \theta_{n^{P}}^{P}(t), \theta_{1}^{C}(t), \ldots, \theta_{n^{C}}^{C}(t)\right)^{\mathrm{T}} .
$$


Remark 7

We note that, by construction, $\mathscr{V} \subset H^{1}(\Omega)$ hence the above lumped model is conforming unlike the one studied in $[8,9]$.

\section{Remark 8}

The mass and stiffness matrices $\tilde{M}$ and $\tilde{A}$ as well as the force vector $\tilde{\mathbf{b}}$, can be easily computed by assembling the ones corresponding to domains $\Omega_{i}, i=1, \ldots, n^{P}+n^{C}$, computed in Section 5 .

Remark 9

All elements in the reduced basis are constant on the ports which can limit the accuracy of the method. This can be overcome in an easy way. Indeed of considering one single reduced basis function associated with each port, we can build a family of them by considering a mesh of the port and then associating to each node a reduced basis function taking the value 1 at this node and 0 on the rest of the nodes on the port.

\section{NUMERICAL RESULTS}

The lumped parameter method, introduced in the previous section for several domains, has been implemented by using Matlab. The program consists of two parts, that can be executed independently:

1. in the first one, a finite element mesh for each domain is loaded; functions $\varphi_{i j}^{P}$ and $\varphi_{i j}^{C}$ are calculated as solution of systems (56)-(59) and (60)-(63); the meshes need not be conforming on the ports, which makes their construction easier;

2. in the second one, function $\tilde{\theta}$ is calculated along the time as solution of (68)-(69), using an implicit Euler method.

The lumped parameter method is suitable for problems where the global mesh is very large and it can be decomposed on smaller pieces. Thus, problems to be solved in the first part are much smaller than the global one and are time independent. Since they can be solved independently, without any information exchange among them, parallelization is straightforward. Moreover, since functions $\varphi_{i j}^{P}$ and $\varphi_{i j}^{C}$ only depend on meshes and functions $k$ and $\alpha_{i}$, they can be calculated only once, even when heating or boundary conditions are time dependent.

The solution of the lumped parameter method has been compared with the solution of a classical finite element method for a real-life engineering application. As a previous step and in order to validate the implementation of the latter, a transient thermal test problem has been solved in the domain $[0,1] \times[0,1.1] \times[0,1.2]$ along the time interval $[0,1]$. A set of meshes $\left\{m_{i}\right\}_{i=3}^{10}$, composed of tetrahedra, has been considered. For $i=3, \ldots, 10$, mesh $m_{i}$ has $i$ intervals along each edge of the domain (see Figure 1). The exact solution of the problem is

$$
u(x, y, z, t)=\cos x \mathrm{e}^{-2 y} \mathrm{e}^{-3 z} t^{3} .
$$

The expected error reduction is quadratic in space and linear in time for the $L^{2}([0, T] ; \Omega)$ norm.

Taking a time step of the type $\Delta t=O\left(h^{2}\right)$, where $h$ is the maximum edge length, we can observe such quadratic decay of the error in the following table (and in Figure 2).

\begin{tabular}{lcc}
\hline Mesh & $\Delta t$ & Error \\
\hline$m_{3}$ & $1.1111 \mathrm{e}-01$ & $2.0080 \mathrm{e}-02$ \\
$m_{4}$ & $6.2500 \mathrm{e}-02$ & $1.1596 \mathrm{e}-02$ \\
$m_{5}$ & $4.0000 \mathrm{e}-02$ & $7.5715 \mathrm{e}-03$ \\
$m_{6}$ & $2.7778 \mathrm{e}-02$ & $5.3369 \mathrm{e}-03$ \\
$m_{7}$ & $2.0408 \mathrm{e}-02$ & $3.9647 \mathrm{e}-03$ \\
$m_{8}$ & $1.5625 \mathrm{e}-02$ & $3.0609 \mathrm{e}-03$ \\
$m_{9}$ & $1.2346 \mathrm{e}-02$ & $2.4339 \mathrm{e}-03$ \\
$m_{10}$ & $1.0000 \mathrm{e}-02$ & $1.9813 \mathrm{e}-03$ \\
\hline
\end{tabular}




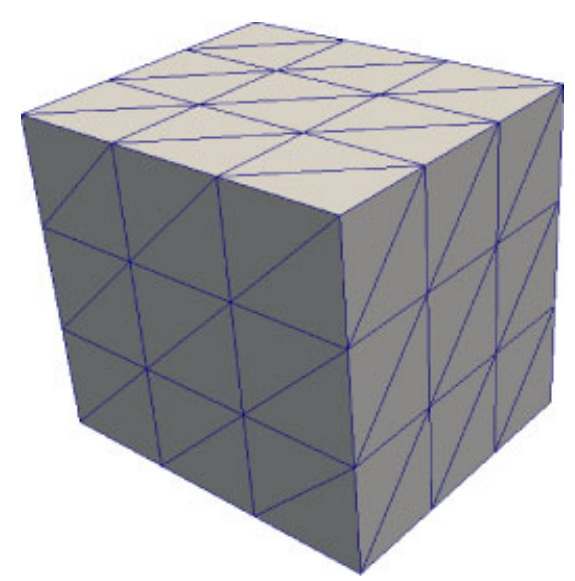

Figure 1. Mesh $m_{3}$ of a parallelepiped (three intervals along each edge).

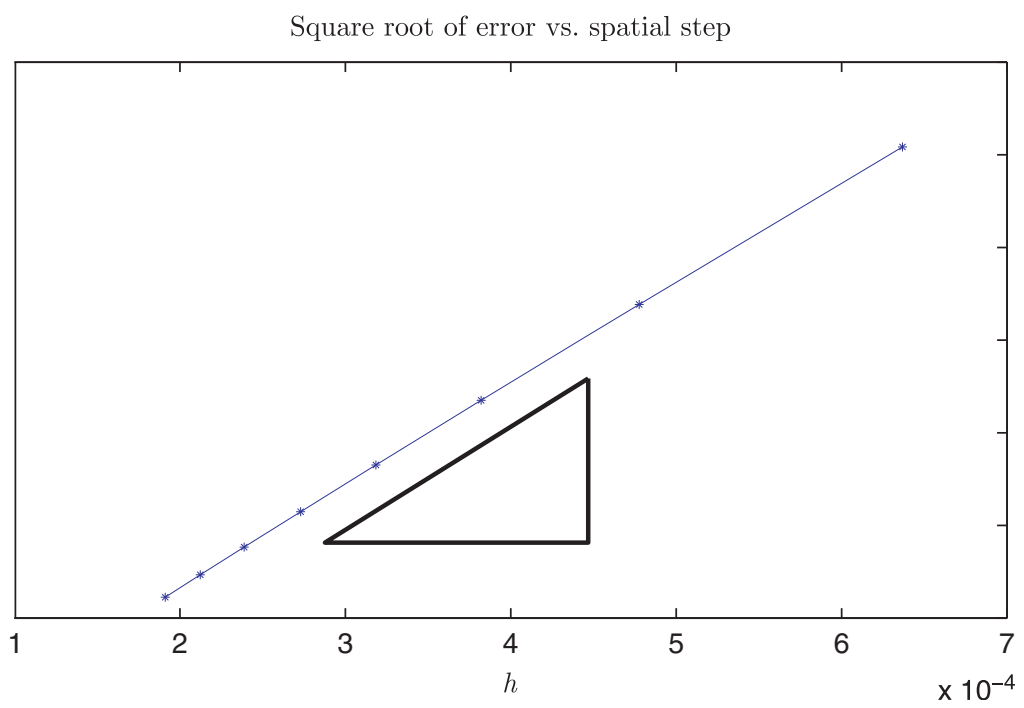

Figure 2. Quadratic decay of the error for the classical finite element approximation.

The comparison between the lumped parameter method and the classical finite element method has been carried out in a 'brushless permanent-magnet' motor, divided into 34 pieces. The electric motor has been designed by the University of Mondragón and the Orona company. The values for electric losses, physical magnitudes and coefficients for boundary conditions have been provided to us by the former institution.

At the beginning of the simulation, the electric motor is at room temperature and it is running for $2 \mathrm{~h}$. Natural convection has been considered outside the motor, while a constant temperature has been taken for air in the inner cavities.

We should note that it is not possible to carry out an error reduction analysis similar to the finite element method. Indeed, theoretically the convergence of the solution of the lumped parameter method would occur when the number of ports tend to infinity. In a realistic case, the number of pieces remains constant, and the only possible improvement can be done in the mesh used to compute the basis functions and/or in the time step. For the purpose of comparison, we created two meshes for the motor:

- the coarse one, composed of 5081 nodes, 21099 elements, and a maximum edge length of $0.04457 \mathrm{~m}$; 

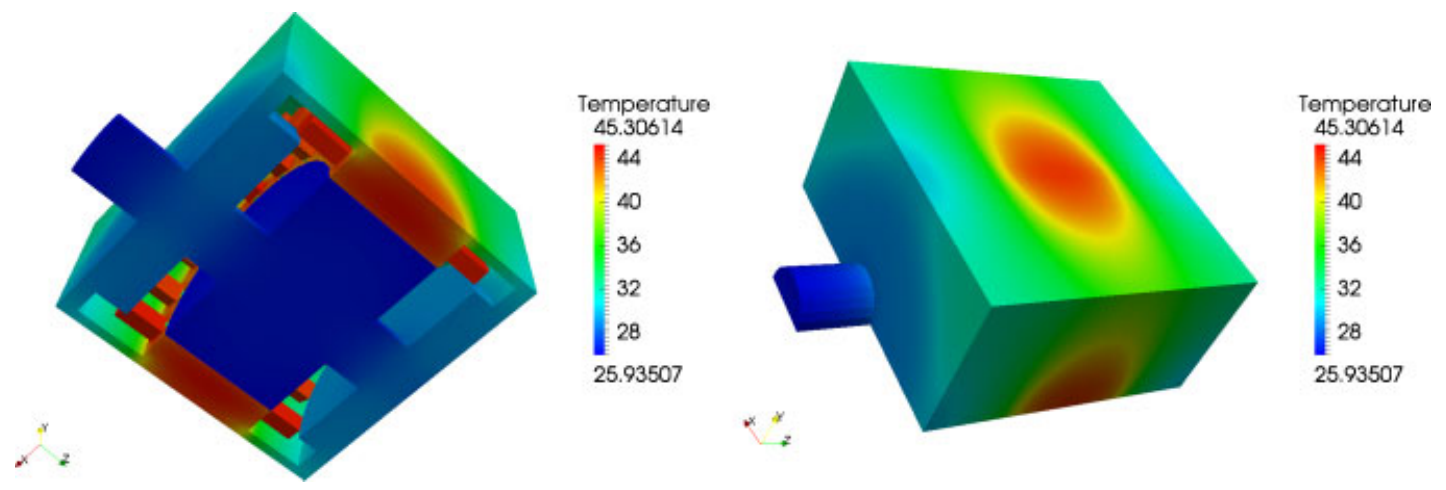

Figure 3. Solution by the finite element method for the thin mesh.
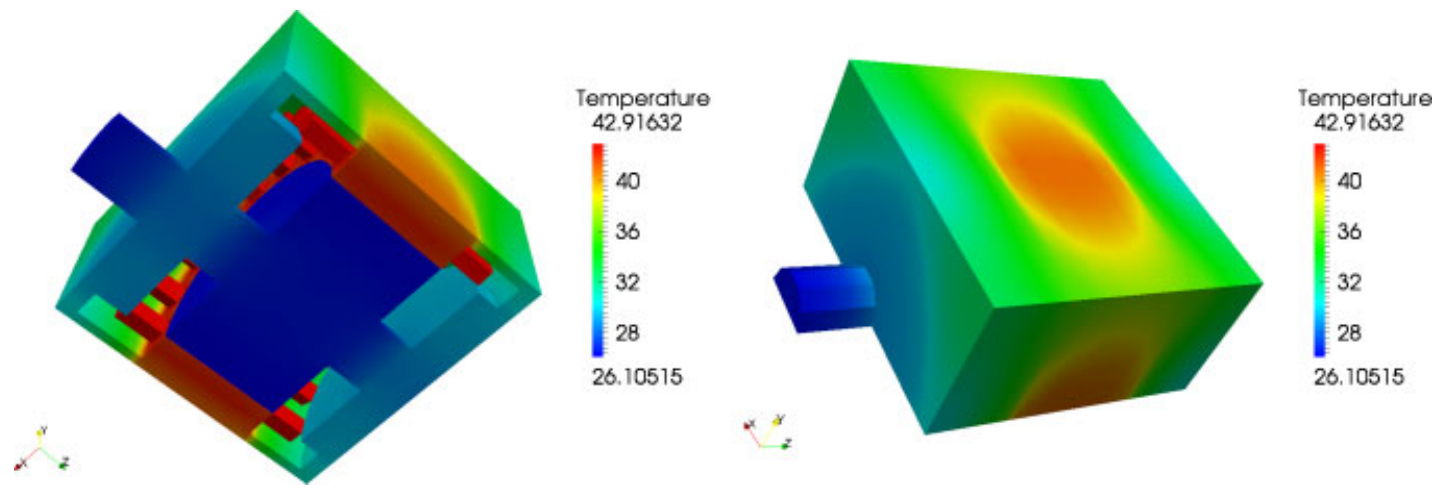

Figure 4. Solution by the lumped parameter method for the coarse mesh.
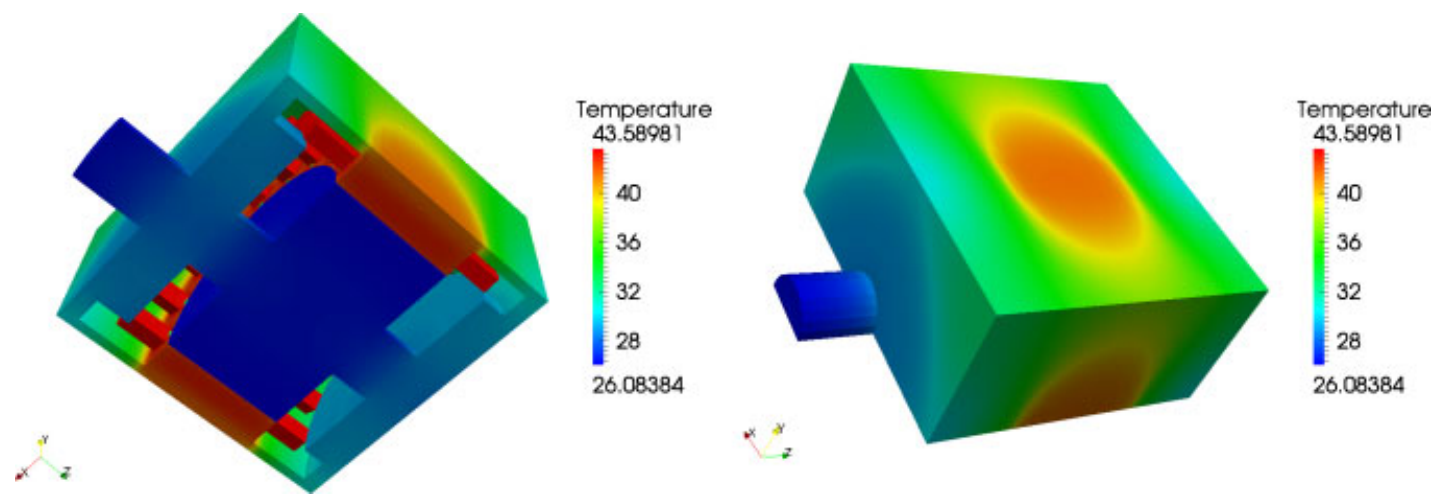

Figure 5. Solution by the lumped parameter method for the fine mesh.

- the fine one, composed of 26066 nodes, 126006 elements, and a maximum edge length of $0.022554 \mathrm{~m}$.

The time step was $10 \mathrm{~s}$.

Figure 3 shows the solution of the finite element method at time $t=2 \mathrm{~h}$, whereas Figures 4 and 5 show the solution of the lumped parameter method at time $t=2 \mathrm{~h}$ for the coarse and the thin meshes, respectively.

First, for the coarse mesh we have compared the solution of problem $\mathbf{P}$ obtained by the finite element method with the one of the lumped parameter model $\mathbf{L P}$ after computing the reduced basis with the same mesh. The relative error in the $L^{2}([0, T] ; \Omega)$ norm was $2.127 \%$. The same 


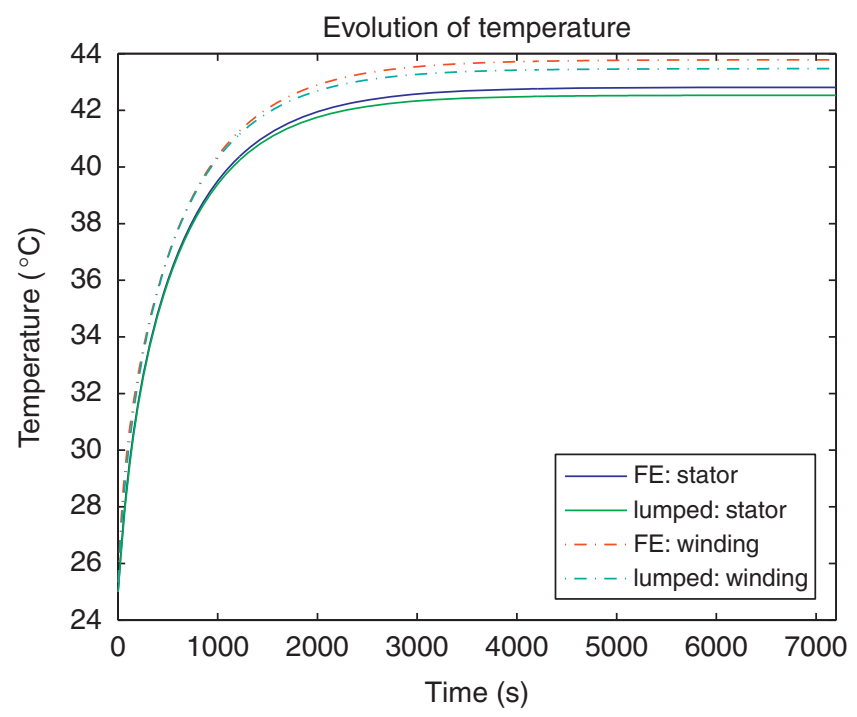

Figure 6. Evolution of temperature in nodes belonging to winding and stator.

computations have been done for the fine mesh. In this case the relative error was $2.056 \%$. We note that the slight improvement should be attributed to a better calculation of the basis functions $\varphi_{i j}^{P}$ and $\varphi_{i j}^{C}$ because we have not increased the dimension of the reduced basis space.

Figure 6 shows the evolution of temperature according to both methods in some nodes, belonging to the winding and the stator, by using the thin mesh.

Of course, the computing time is favorable to the lumped parameter method. For the thin mesh, the first part of the computations lasted $275 \mathrm{~s}$ (the basis was computed sequentially) and for the second part it lasted only $83 \mathrm{~s}$. We took advantage of the fact that air temperature $\tilde{\theta}_{i}^{C}$ and heating $f$ were time independent, allowing to precalculate a part of vector $\tilde{b}$ in (70). The computing time for the finite element method was $715 \mathrm{~s}$. We note that a mesh refinement used in the first part does not involve a longer computing time in the second part, since the degrees of freedom for the latter are only related to the number of ports and convective boundaries.

\section{CONCLUSIONS}

We have introduced a family of lumped parameter models for solving parabolic partial differential equations as those arising from transient heat transfer problems. The approach consists of a Galerkin method corresponding to reduced bases defined for a domain decomposition. These bases are well adapted to the geometry and the physics of the problem hence good approximations can be obtained with a very low number of degrees of freedom.

One of the advantages of the method with respect to the lumped parameter methods existing in the bibliography is that the capacitance and resistance matrices are built in a general and automatic way hence we are not obliged to introduce any geometrical parameters which are, in general, rather arbitrary.

\section{ACKNOWLEDGEMENTS}

The authors express their gratitude to Dr Javier Poza and Gaizka Almandoz from the Universidad de Mondragón (Spain) and to Antonio González from ORONA company for attracting their attention to the lumped parameter models and for providing the data for the numerical experiments.

The authors also thank the referees for providing helpful suggestions and remarks on the previous version of this paper. 


\section{REFERENCES}

1. Mellor H, Roberts D, Turner R. Lumped parameter thermal model for electric machines of TEFC design. IEE Proceedings on Industry Application 1991; 138(5):205-218.

2. Boglietti A, Cavagnino A. Staton D, Shanel M, Mueller M, Mejuto M. Evolution and modern approaches for thermal analysis of electrical machines. IEEE Transactions on Industrial Electronics 2009; 56(3):871-882.

3. Boglietti A, Cavagnino A, Lazzari M. A simplified thermal model for variable-speed self-cooled industrial induction motor. IEEE Transactions on Industry Application 2003; 39(4):945-952.

4. Tang WH, Wu QH, Richardson ZJ. A simplified transformer thermal model based on thermal-electric analogy. IEEE Transactions on Power Delivery 2004; 19(3):1112-1119.

5. Gerling D, Dajaku G. Novel lumped parameter thermal model for electric machines. Technical Report 11-2004, Institute for Electrical Drives, University of Federal Defense Munich, 2004.

6. Lassila T, Rozza G. Parametric free-form shape design with PDE models and reduced basis method. Computer Methods in Applied Mechanics and Engineering 2010; 199:1583-1592.

7. Kunisch K, Volkwein S. Galerkin proper orthogonal decomposition methods for parabolic problems. Numerische Mathematik 2001; 90(1):117-148.

8. Maday Y, Ronquist M. A reduced-basis element method. Journal of Scientific Computing 2002; 17(1-4):447-459.

9. Maday Y, Ronquist M. The reduced basis element method: application to a thermal fin problem. SIAM Journal of Scientific Computing 2004; 26(1):240-258.

10. Grepl MA, Maday Y, Nguyen NC, Patera AT. Effcient reduced-basis treatment of nonaffine and nonlinear partial differential equations. Modélisation Mathématique et Analyse Numérique 2007; 41(3):575-605.

11. Boyaval S, Le Bris C, Maday Y, Nguyen NC, Patera AT. A reduced basis approach for variational problems with stochastic parameters: application to heat conduction with variable Robin coefficient. Computer Methods in Applied Mechanics and Engineering 2009; 198(41-44):3187-3206.

12. Zienkiewicz OC, Taylor RL. The Finite Element Method, Vol. 1. The Basis. Butterworth-Heinemann: Oxford, 2000.

13. Quarteroni A, Valli A. Numerical Approximation of Partial Differential Equations. Springer: Berlin, Heidelberg, 2008.

14. Toselli A, Widlund O. Domain Decomposition Methods: Algorithms and Theory. Springer: Berlin, Heidelberg, 2005. 